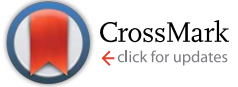

Cite this: RSC Adv., 2015, 5, 70005
Received 19th May 2015

Accepted 5th August 2015

DOI: $10.1039 / \mathrm{c} 5 \mathrm{ra09377e}$

www.rsc.org/advances

\section{Micelle stability in water under a range of pressures and temperatures; do both have a common mechanism? $\dagger$}

\begin{abstract}
Yanis Ricardo Espinosa Silva and J. Raul Grigera*
The formation of sodium dodecylsulfate (SDS) micelles in water and heavy water at different pressures and temperatures using molecular dynamics simulations was used to analyze their stability and structure under different conditions and to evaluate the agreement with existing experiments. The results show the assembling of micelles at 1 bar and the presence of larger aggregates under high pressure (over $3 \mathrm{kbar}$ ). These large aggregates are not micelles but small finite pieces of bilayers in rod-like shapes. The results obtained using systems at different temperatures showed that both high and low temperatures produce lamellar structures. It is well-known that micelles expose polar residues to water and leave non-polar residues inside where they interact via hydrophobic interactions. High pressure as well as low and high temperatures inhibit the hydrophobic interactions, and under these conditions other structures are produced instead of micelles. This process seems to be similar to protein denaturation under certain temperatures and pressures.
\end{abstract}

\section{Introduction}

The stability of the aggregates of amphiphilic surfactants in water at different pressures and temperatures has been widely discussed in the literature. ${ }^{1-10}$ The results obtained using various experimental techniques (conductivity, light scattering, dynamic fluorescence probing, and small-angle neutron scattering) indicate that micelles are formed in an aqueous solution of surfactants at 1 bar when the concentration of sodium dodecylsulfate (SDS) (see Fig. 1) reaches the critical micelle concentration (CMC). With increasing pressure, the aggregation number decreases and at around 1 kbar the micelles are disassembled. At higher pressures, large aggregates appear which was interpreted as the re-entrance of the micellar phase with larger aggregate numbers. It is widely accepted that hydrophobic interactions are the driving force for the assembly of micelles but we have to be aware that at pressures above 1 kbar, water changes the structure of the micelles, decreasing the ratio of low/high water structures, causing hydrogen bond lattice weakening, or even eliminating the hydrophobic interactions. This fact seems to contradict the possibility of large micelles at high pressures. Recently, Baltasar $e t$ al. ${ }^{6}$ have used a sound speed technique on a sodium dodecanoate-water solution under pressure and their results suggest that a gel-like phase is formed above $2.5 \mathrm{kbar}$. We cannot ignore the

CEQUINOR, University of La Plata and CONICET, 47 y 115, B1900 La Plata, Argentina.E-mail: raul@grigera.com.ar

$\dagger$ Electronic supplementary information (ESI) available. See DOI: 10.1039/c5ra09377e experimental evidence for the existence of large aggregates at high pressures. Thus, with the aim of contributing to the explanation of the apparent discrepancy regarding the nature of the properties of the water-surfactant mix under pressure, we studied the behaviour of a solution of SDS in water and heavy water at different pressures and temperatures using molecular dynamics simulation.

\section{Computational methods}

The systems under study were a mixture of sodium dodecylsulfate in water or heavy water. The model of SDS used was developed by Sammalkorpi and co-workers ${ }^{7}$ (see Fig. 1). For water, we used the Simple Point Charge Extended (SPC/E) model ${ }^{11}$ and for heavy water the Simple Point Charge Heavy Water (SPC/HW) model. ${ }^{12}$ Molecular Dynamics (MD) simulations were done using the GROMACS 4.5.3 package, ${ }^{13}$ the equations of motion were solved using a leap-frog integration step. We used a cubic simulation box, with periodic boundary conditions. The systems were initially equilibrated with an NPT ensemble at $300 \mathrm{~K}$ and $1 \mathrm{bar}$ and then the required temperature

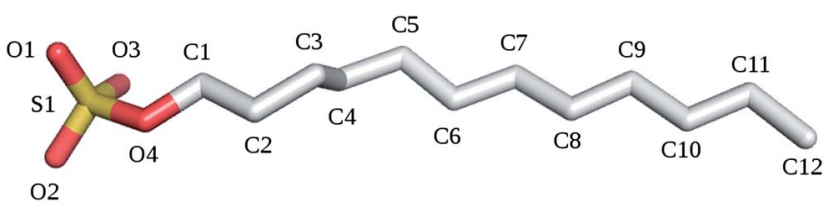

Fig. 1 The sodium dodecylsulfate molecule employed in our MD simulations with the reference numbers of each atom. 
and pressure were fixed using a weak coupling with the Vrescale thermostat ${ }^{14}$ and the Berendsen barostat ${ }^{15}$ with coupling time constants of $0.1 \mathrm{ps}$ and $1.0 \mathrm{ps}$, respectively. The Lennard-Jones (LJ) interactions and long-range electrostatic interactions were cut off at $1.2 \mathrm{~nm}$ and to calculate long-range electrostatic interactions we use the reaction field method. ${ }^{\mathbf{1 6}}$ Throughout the simulations the integration time step was 2 fs. Water molecules and SDS were constrained using the LINCS algorithm. ${ }^{17}$

\section{Systems under pressure}

The simulations started with a random distribution of the SDSwater mixture at a concentration above the CMC. We first studied the behaviour of the self-aggregation of SDS in the SPC/ E model and the SPC/HW model, varying the number of SDS molecules and the water molecules were kept at a constant concentration (500 mM). Thus, 70 and 200 SDS molecules were hydrated with 6667 and 16792 solvent molecules, respectively. The configurations that resulted after the self-aggregation process were used as the starting points for the simulations under pressure. After the equilibrium was reached at $1 \mathrm{bar}$, the system was coupled to a barostat, increasing the pressure at regular intervals up to 4000 bar.

For each selected pressure, the systems of 70 and 200 SDS were simulated for $10 \mathrm{~ns}$ and $100 \mathrm{~ns}$, respectively. The data was collected after equilibration for $5 \mathrm{~ns}$ and $50 \mathrm{~ns}$, respectively.

\section{Cluster analysis}

Cluster analysis was done using Stillinger's direct connectivity criterion, ${ }^{18}$ where the connecting time is not required, instead the connectivity is checked for each frame, i.e., this criterion is based solely upon the distance between the particles. Therefore, two molecules are considered members of a cluster when the distance between the C6-carbon atoms is less than or equal to $3 /$ 2 of the diameter $(\sigma)\left(R_{\text {cut }}=0.60 \mathrm{~nm}\right)$. The molecules were selected using the Stoddard algorithm. ${ }^{19}$

We can define probability, $P_{s}$, to find a cluster of $s$ monomers as: ${ }^{20}$

$$
P_{s}=\frac{n(s) s}{N}
$$

where $n(s)$ is the number of aggregates of $s$ monomers and $N$ is the total number of monomers. The ratio $n(s) / N$ can be considered as a relative frequency, thus $P_{s}$ satisfies the normalization condition.

$$
\sum_{s=1}^{N} P_{s}=1
$$

The aggregation process can also be analyzed using the radial distribution function, $g(r)$. For all pairs of SDS molecules, we plotted the first peak of the radial distribution function between the $\mathrm{C} 1$ and $\mathrm{C} 12$ carbon atoms against pressure. The SDS solvent-accessed surface was calculated using the g_sas algorithm ${ }^{13}$ with a probe radius of $1.4 \AA$.

\section{Results and discussion}

\section{Self-assembly}

As we stated before, the criteria adopted to define the formation of micelles were based on a geometrical classification of the distance between the C6-atoms of all SDS molecules. The solutions at the desired concentration $(500 \mathrm{mM})$ were randomly distributed in a simulation box of arbitrary size and the simulation was started at 1 bar and $300 \mathrm{~K}$. After equilibration, a longer run was carried out to collect enough data. Fig. 2 shows the normalized cluster size against the aggregation number for $100 \mathrm{~ns}$ of simulation following equilibration.

The aggregation process was monitored over time by recording the area of SDS exposed to the solvent. Fig. 3 corresponds to a system of 200 SDS monomers and 16972 SPC/E water molecules or SPC/HW heavy water molecules, respectively ( $500 \mathrm{mM}$ for both systems) at $300 \mathrm{~K}$ and 1 bar. We can see that the micelles were formed at around the first $20 \mathrm{~ns}$. For $\mathrm{D}_{2} \mathrm{O}$, the final exposed area was lower than that for $\mathrm{H}_{2} \mathrm{O}$. This difference was in agreement with the slightly stronger energy of the D-bond compared with the H-bond.

The maximum aggregation number in the system of 70 molecules in the SPC/E model was 62 SDS molecules. For the SPC/HW model at the same concentration, the formed micelles had a size of between 65 to 67 monomers. It should be noted that the temperature was set slightly higher than the critical micellization temperature (CMT) reported for SDS. ${ }^{21}$

To test whether the results depend on the system size, we have built a system of 200 monomers at $500 \mathrm{mM}$. The results show that there are no significant differences in micellization between the systems when increasing the system size (see Fig. S1 and S2, in ESI $\dagger$ ).

\section{Micelles under pressure}

We studied the effects of pressure on the micelles already formed by analyzing the phase transition between the states at 1 bar and 4000 bar at $T=300 \mathrm{~K}$. The structures were evaluated by plotting the first peak of the radial distribution function of the carbon atoms $\left(\mathrm{C}_{1}-\mathrm{C}_{12}\right)$ (see Fig. 1) against the pressure.

To describe the structural changes of the micelles at different pressures, we computed the average number of clusters $\left(N_{\text {clust }}\right)$ and the average aggregation number $\left(N_{\text {agg }}\right)$ for a

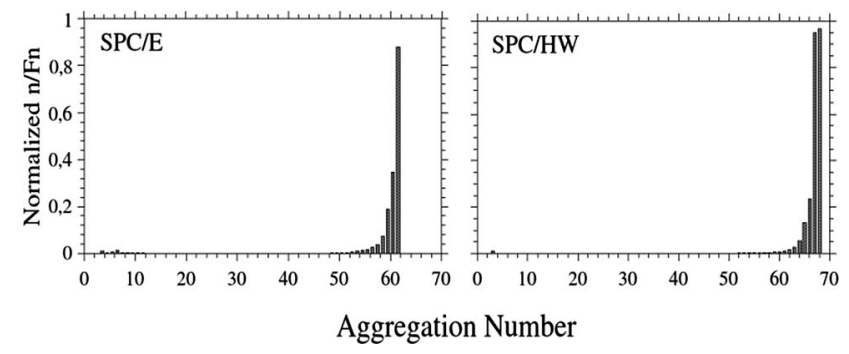

Fig. 2 The maximum micellar aggregation numbers in a system with 70 SDS molecules. A normalized distribution of cluster size against the aggregation number of SDS in water (SPC/E) and heavy water (SPC) $\mathrm{HW})$ at $500 \mathrm{mM}$. 


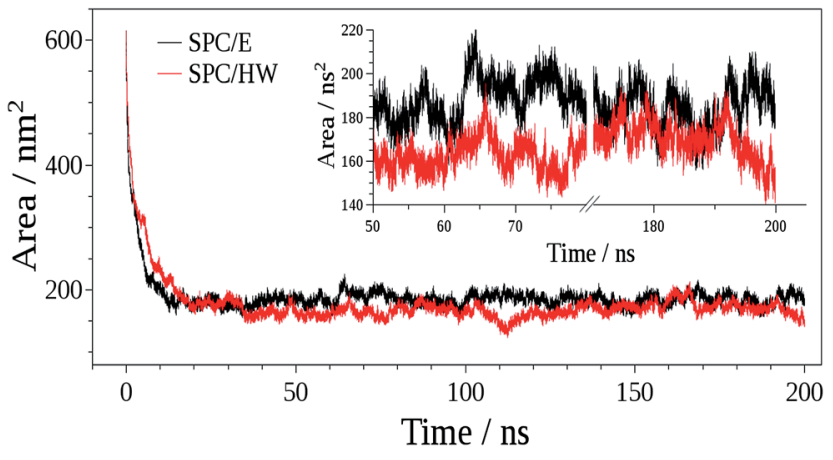

Fig. 3 Changes over time of the area of SDS exposed to the solvent in water (black) and heavy water (red). The inset shows a zoomed in section of the area of SDS exposed to the solvent.

range of pressures between 1 bar and 4 kbar in the systems of 70 SDS and 200 SDS monomers. A version with a solvent for the SPC/E water model and SPC/HW heavy water model was simulated for each of those systems.

Fig. 4 shows the changes in $N_{\text {agg }}$ and $N_{\text {clust }}$ at different pressures in the system of 70 SDS monomers. We can observe that in water the average aggregation number, $N_{\text {agg }}$ (black lines), starts to decrease at around 1 kbar and reaches a minimum at around 1400 bar. From that point, a sharp increase is observed, reaching a large aggregation number. Accordingly, the number of clusters, $N_{\text {clust }}$, follows the opposite trend (red lines), close to 1200 bar $N_{\text {clust }}$ displays the maximum value and starts to diminish until 2200 bar. In heavy water, we observed that although demicellization kinetics seem to have the same trend as in water, nevertheless in the SPE/HW model the high energy of the D-bond makes it more difficult for the micelles to disassemble and the formation of smaller aggregates results.

This behaviour was experimentally observed via light scattering ${ }^{\mathbf{1}}$ and interpreted as a partial disassembling at around 1 kbar, with the formation of a larger number of micelles of smaller size at a lower pressure, and at a larger pressure, a decrease in the number of micelles accompanied by an increase in size.

The present results are consistent with the experiments, since at 1 bar we observed a number of aggregates (micelles)

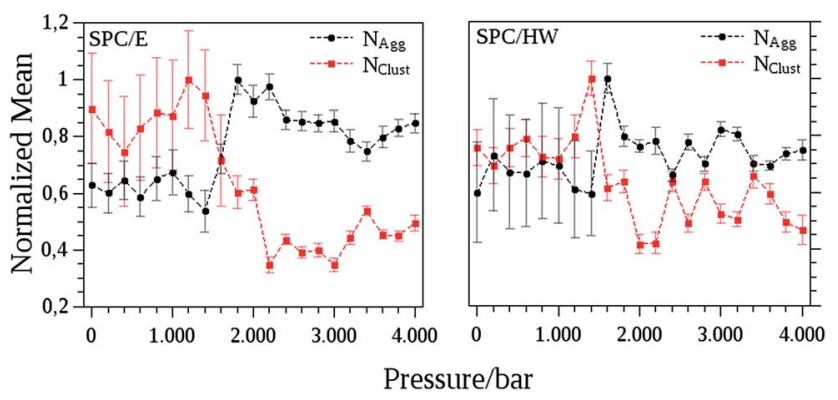

Fig. 4 The normalized cluster size ( $N_{\text {clust, }}$ red) and normalized aggregation number $\left(N_{\text {agg, }}\right.$, black) for water (SPC/E) and heavy water (SPC/HW). Dots and error bars represent the average and the standard error, respectively. which were at least partially segregated (a large number of small clusters) when the pressure was increased. At pressures above but close to 1 kbar, we found a lower number of larger aggregates. When the pressure was increased, larger lamellar-like aggregates were produced. We have to bear in mind that the number of clusters computed using this method includes elements of quite different sizes. However, we cannot conclude that we have large micelles present at high pressures.

Fig. 5 shows a visualization of the process of the changes in the aggregation under pressure following the radial distribution function, $g(r)$, normalized for $\mathrm{C}_{1}-\mathrm{C}_{12}$ (see sec Cluster analysis) in water and heavy water with 200 SDS molecules. This alternative representation also shows that the micellar phase was stable at 1 bar, but the increase of pressure generated a transformation process that, above $2 \mathrm{kbar}$, led to larger aggregates.

Fig. 6 shows another way of depicting the process by plotting the area of SDS exposed to the solvent. At the bottom of the figure we can see the images taken from the simulation that show the behaviour of the aggregation of SDS at three different pressures: micelles at 1 bar, partially disassembled aggregates at 1500 bar, and lamellar structures at 4000 bar. It is known that at 1 bar, amphiphilic surfactants such as SDS which have welldefined polar heads and hydrophobic tails tend to form spherical micelles. The polar heads interact with the counterions of the solution and the hydrophobic tails are oriented towards the centre, forming sphere-like micelles.

When the pressure was increased to $1500 \mathrm{bar}$, the water began to lose its tetrahedral structure, weakening the hydrophobic interactions; ${ }^{22,23}$ the internal micellar core was thus at least partially exposed to the solvent, causing disassembling of the micelles. With a further increase in pressure (4000 bar), large structures were observed that were interpreted as micelles of larger dimensions than those observed at 1 bar. ${ }^{1}$

At first glance, the image seems to show a lamellar structure. However, Fig. 7, which shows the picture corresponding to 4000 bar from two different views, seems to indicate that the lamellar phase does not actually consist of extended stacked bilayers but

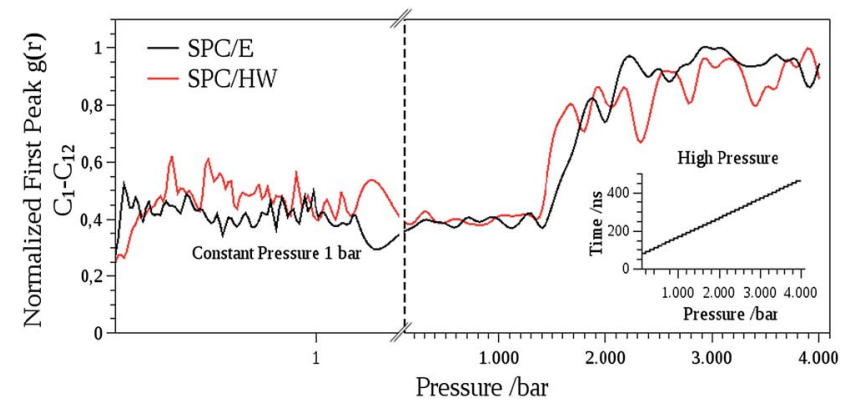

Fig. 5 The normalized first peak of the radial distribution function of the SDS-water and SDS-heavy water systems along the simulation run at different pressures. This representation is consistent with the results of the number of aggregates at different pressures. Normalization was performed using the ratio $g(r)_{0} / g(r)_{\max }$, where $g(r)_{0}$ is the most probable distance between the neighbouring atoms and $g(r)_{\max }$ is the maximum probable distance between the neighbouring atoms (non-standardized data in Fig. S3 of ESI†). 


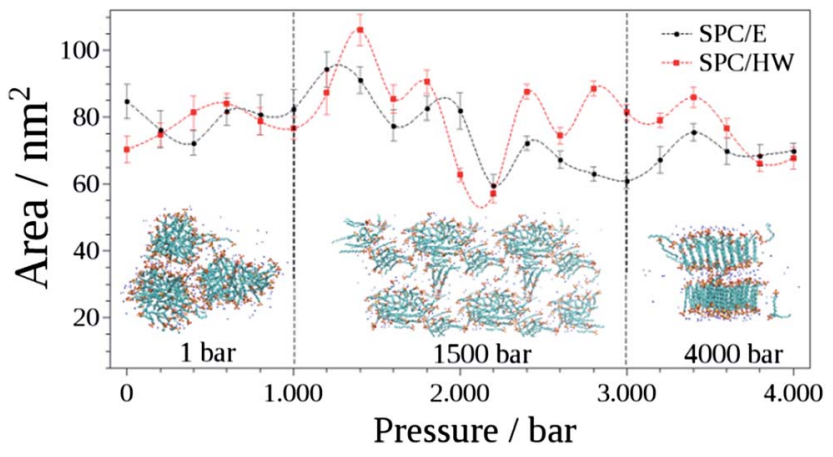

Fig. 6 Exposed areas of the 200 SDS monomers in water (black line) and heavy water (red line) at different pressures. The images of the aggregates were obtained from the simulation. Dots and error bars represent the average and the standard deviation, respectively.

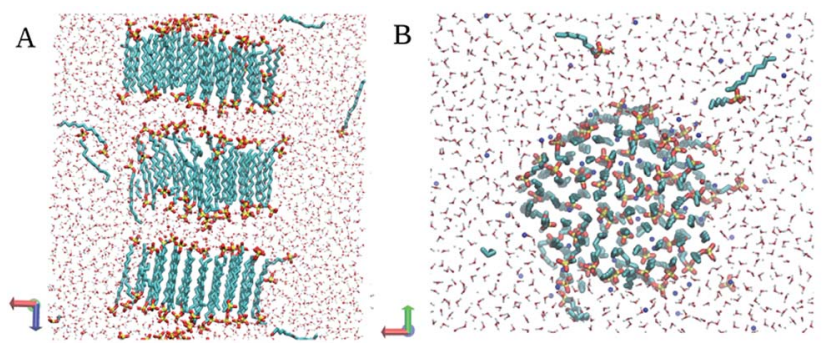

Fig. 7 Structures of the aggregates at 4000 bar. Picture A suggests small finite pieces stacked in a lamellar structure. However, considering picture $\mathrm{B}$ (rotated 90 degrees compared to A) a disk is evident and the structure is a stacked disk rod. The system in this example is observed with 200 SDS molecules, but the change in the structure is the same in both systems.

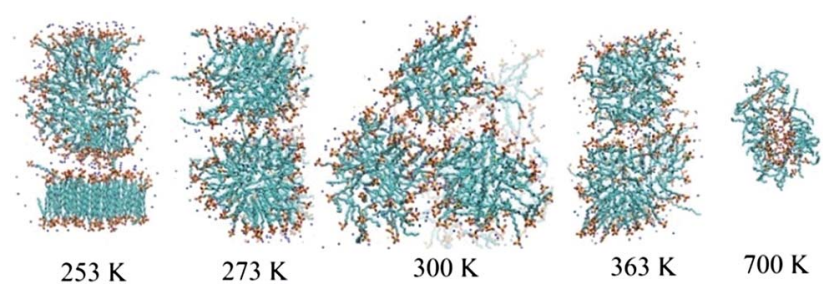

Fig. 8 Structures of 200 SDS molecules in water at five different temperatures.

rather of layers of small finite pieces of bilayers (e.g., disks and ribbons). ${ }^{24}$ In our simulations we see that the change in the geometrical aggregation is consistent with a stacked disk rod in the lamellar phase. This result agrees with Chen and Ruckenstein, ${ }^{25}$ who reported about the effect of the selectivity of the solvent in changing morphologies of copolymer aggregation, using a dissipative particle dynamics (DPD) simulation. Likewise, Baltasar et al. ${ }^{6}$ noted in an experimental study that the changes in the aggregation states of surfactants can be explained in terms of the effect of pressure on the characteristics of the hydrogen bonding network of water, favoring the appearance of lamellar phases.

\section{Temperature effects}

The contribution to the free energy of the hydrophobic interactions has an entropic origin, therefore, if the temperature decreases then the contribution of the entropic term, $-T \Delta S$, will decrease too. Thus, we expected that lowering the temperature of a system of SDS in water would have a similar effect as increasing the pressure. On the other hand, high temperature increases the entropy of the system but the aggregation of nonpolar regions will not produce additional entropy (the driving force of hydrophobic interactions) and as a consequence, we expected aggregates based mainly on polar interactions. To check the hypothesis, we performed a molecular dynamics simulation of a system of SDS using the SPC/E model in water already stabilized at different temperatures. Fig. 8 shows the change in the structure of a system already equilibrated at 1 bar at temperatures from 253 to $700 \mathrm{~K}$.

At the analyzed low temperature $(253 \mathrm{~K})$, we observed an aggregate that seemed to evolve to a stacked disk rod structure too. The effect of low temperature seemed to be similar to the pressure effect. The image at $273 \mathrm{~K}$ shows an intermediate state. At $300 \mathrm{~K}$, we observed regular micelle formation. When the temperature increased to $363 \mathrm{~K}$, the structure changed, showing a large aggregate composed of two similar large interacting aggregates but more disordered than the one observed at $253 \mathrm{~K}$, when the formation of the stacked disk rod lamellae was in progress. An inverse micelle was observed at $700 \mathrm{~K}$. This structure was due to the fact that at such high temperature, water is in the vapour phase and the polar interactions produced an inverse micelle shape.

\section{Conclusions}

The results obtained using simulations showed that SDS micelles in water were disassembled when the pressure increased up to $1 \mathrm{kbar}$. At higher pressures, a rearrangement process was observed, producing stacked disk rod structures. The changes at high pressures can be explained by considering the changes in water under pressure from a predominantly hexagonal structure, low-density (LD), to a predominantly tetrahedral one, high-density (HD). These structural changes result in a process of gradual inhibition of the hydrophobic interactions when the pressure increases. Micelles are formed in SDS at normal pressures due to the association of the nonpolar tail, exposing the polar head to the solvent neutralized by the counter-ions. At around 1 kbar, the hydrophobic interaction was weaker, allowing the exposure of the non-polar tails. With a further increase of pressure, the system was compacted. Non-polar tails can associate via a weak attraction, exposing their surface to the solute without restriction. At the same time, the polar heads associate with each other with the help of counter-ions, producing a compact structure with a lamellar shape. It was experimentally shown that large aggregates are formed at high pressures, which are compatible with the stacked disk rod shape shown by the simulation results.

Regarding the effect of the temperature, we observed that both low and high temperatures produced similar structures as 
at high pressures. This is understood since the hydrophobic interaction depends on the entropic contribution of the free energy $(-T \Delta S)$. Therefore, at high and low temperatures, the association of the non-polar solutes will not increase the entropy through the association of the non-polar residues, producing different structures. This process is similar to the temperature and pressure denaturation of proteins. ${ }^{26}$

Due to the similitude of the changes in both systems, we might name the phenomenon of the micelle structure at low temperatures and high pressures as micelle denaturation.

\section{Acknowledgements}

We wish to thank Dr Ernesto Caffarena for his interesting comments and Dr Ariel Alvarez for his help with MD simulations. The work was funded by the National Council of Argentina (CONICET) and the University of La Plata (UNLP); Y. R. Espinosa was supported by the CONICET and J. R. Grigera is a member of the Research Career of the same institution and an Emeritus Professor of the UNLP.

\section{References}

1 N. Nishikido, M. Shinozaki, G. Sugihara, M. Tanaka and S. Kaneshina, J. Colloid Interface Sci., 1980, 74, 474.

2 K. Hara, N. Baden and O. Kajimoto, J. Phys.: Condens. Matter, 2004, 16, S1207.

3 J. S. Collura, D. E. Harrison, C. J. Richards, T. K. Kole and M. R. Fisch, Langmuir, 2001, 17, 8084.

4 R. de Lisi, S. Milioto and N. Muratore, J. Phys. Chem. B, 2001, $105,4846$.

5 S. Kaneshina, M. Tanaka, T. Tomida and R. Matuura, $J$. Colloid Interface Sci., 1974, 48, 450.

6 E. H. Baltasar, M. Taravillo, P. Sanz, D. Baonz and B. Guignon, Langmuir, 2014, 30, 14743-47477.

7 M. Sammalkorpi, M. Karttunen and M. Haataja, J. Phys. Chem. B, 2007, 111, 11722-11733.
8 K. Hara, H. Suzuki and N. Takisawa, Phys. Chem., 1989, 93, 3710-3713.

9 A. Jusufi, S. A. Sanders, M. L. Klein and A. Z. Panagiotopoulos, J. Phys. Chem. B, 2011, 115, 990-1001.

10 S. A. Sanders, M. Sammalkorpi and A. Z. Panagiotopoulos, J. Phys. Chem. B, 2012, 116, 2430-2437.

11 H. J. C. Berendsen, J. R. Grigera and T. P. Straatsma, J. Phys. Chem., 1987, 91, 6269-6271.

12 J. R. Grigera, J. Chem. Phys., 2001, 114, 8064-8067.

13 D. van der Spoel, E. Lindahl, B. Hess, G. Groenhof, A. E. Mark and H. J. Berendsen, J. Comput. Chem., 2005, 26, 1701-1718.

14 G. Bussi, D. Donadio and M. Parrinello, J. Chem. Phys., 2007, 126, 014101-014107.

15 H. J. Berendsen, J. P. M. Postma, W. F. vanGunsteren, A. R. H. DiNola and J. R. Haak, J. Phys. Chem., 1984, 81, 3684-3690.

16 I. G. Tironi, R. Sperb, P. E. Smith and W. F. A. vanGunsteren, J. Chem. Phys., 1995, 102, 5451-5459.

17 B. Hess, H. Bekker, H. J. Berendsen and J. G. Fraaije, J. Comput. Chem., 1997, 18, 1463-1472.

18 F. H. Stillinger, J. Chem. Phys., 1963, 38, 1486-1494.

19 S. D. Stoddard, J. Comput. Phys., 1978, 27, 291-293.

20 D. Stauffer and A. Aharony, in Introduction to Percolation Theory, Taylor \&Francis, London, 2nd edn, 1994, pp. 15-60.

21 S. Paula, W. Sues, J. Tuchtenhagen and A. Blume, J. Phys. Chem., 1995, 99, 11742-11751.

22 O. Chara, A. N. McCarthy and J. R. Grigera, Phys. Lett. A, 2011, 375, 572-576.

23 C. G. Ferrara and J. R. Grigera, Chem. Phys., 2014, 434, 15-19.

24 W. M. Gelbart and A. Ben-Shaul, J. Phys. Chem., 1996, 100, 13169-13189.

25 (a) H. Chen and E. Ruckenstein, Soft Matter, 2012, 8, 89118916; (b) H. Chen and E. Ruckenstein, Soft Matter, 2012, 8, 1327-1333.

26 C. L. Dias, T. Ala-Nissila, J. Wong-ekkabut, I. Vattulainen, M. Grant and M. Karttunen, Cryobiology, 2010, 60, 91-99. 